\title{
Edaphic Drivers of Community Structure and Composition in a Mixed Ombrophilous Forest
}

\author{
Juliano Cordeiro ${ }^{1}$ (i) 0000-0001-8047-7463 \\ Victor Pereira Zwiener ${ }^{1}$ (D) 0000-0003-1693-8960 \\ Gustavo Ribas Curcio² (1) 0000-0002-6426-9741 \\ Carlos Vellozo Roderjan ${ }^{1}$ (i) 0000-0002-1872-5355
}

\begin{abstract}
This study sought to assess the variation in species composition and community structure considering soil characteristics. Based on physical and chemical analyses of soil, we selected four sites for vegetation sampling: Haplic Cambisol (P1), Regolithic Neosol (P2), Latosol Bruno (P3) and Humic Cambisol (P4). In each site, we sampled all arboreal individuals with $\mathrm{DBH} \geq 4.8 \mathrm{~cm}$. Sites were distinguished by species with different values of importance and indicator index (indVal). Sites were clearly separated in biplots generated by canonical ordination (RDA), which indicated that the distribution of species correlates with acidity, $\mathrm{Al}+, \mathrm{C}$ and $\mathrm{P}$ concentrations, $\mathrm{CEC}, \mathrm{pH}$, base saturation, drainage, and pedological unit. Our results show that local scale variation in soil attributes influences tree species composition and community structure, and, thus, should be considered for understanding the distribution, occupation, and response of tree species in remnants of Mixed Ombrophilous Forest.
\end{abstract}

Keywords: Atlantic Forest, environmental gradients, forest inventory, multivariate analysis, soil-vegetation relationship.

\section{INTRODUCTION AND OBJECTIVES}

Identifying environmental drivers that regulate the coexistence of species and their respective abundances is an important step towards the understanding of how plant communities are organized and assembled (Reinhart, 2012). Environmental feedbacks act at different spatial scales and can limit or enhance the dominance of plant species (Lortie et al., 2004). At local scales, environmental conditions affect competitive outcomes and the extension of community structure (Lortie et al., 2004; Reinhart, 2012). The Mixed Ombrophilous Forest (MOF) has the largest geographical distribution area in the southern Brazilian states, with the conifer Araucaria angustifolia (Bertol.) Kuntze (Araucariaceae), which is considered the species that most characterizes this forest type (IBGE, 2012). Despite the wide range of environmental conditions in the MOF, little is known about factors that determine plant community structure and floristic variation at local scales in this phytogeographical region.

The MOF is part of the Atlantic Forest biome, a highly threatened biodiversity hotspot (Mittermeier et al., 2011).
These forests once occupied 37\% of Paraná state in Southern Brazil; however, the MOF original extent has been reduced and fragmented due to the intense exploitation of natural resources, expansion of agriculture and urbanization. Data from 2004 shows that most remaining fragments are highly impacted with only $0.8 \%$ (66,000 ha) of well-preserved vegetation (Castella \& Britez, 2004). MOF remnants are found in different stages of ecological succession and conservation, scattered across the three plateaus of the state of Paraná and in small forest patches of southern-southeastern Brazil (FUPEF, 2001). These MOF remnants face different environmental conditions that characterize its range of occurrence. Such variation in regional and local environmental variables is expected influence mechanisms that determine how communities are structured, with emphasis on local edaphic variables (Sollins, 1998).

The relationships between the distribution of tree species and community composition with different pedological, topographic and climate variables have been characterized in multiple studies across the Atlantic Forest biome. However, most previous studies have been conducted in tropical wet and seasonal forest typologies

\footnotetext{
${ }^{1}$ Universidade Federal do Paraná (UFPR), Curitiba, PR, Brasil

${ }^{2}$ Empresa Brasileira de Pesquisa Agropecuária (Embrapa), Colombo, PR, Brasil
} 
or at broad scales, lacking detailed information required to assess variation at local communities (Camargos et al., 2008; Marcilio-Silva et al., 2017; Oliveira-Filho et al., 2001; Pinto et al., 2005). In the context of the MOF, studies seeking to assess relationships between vegetation and environmental variables, such as Vibrans et al. (2008) and Carvalho et al. (2009), need to be practiced by a larger number of researchers if we aim to achieve a general understanding of how different environmental conditions affect floristic composition and community structure of this vegetation type. Knowledge about drivers of floristic differentiation, species abundance and distribution is an essential information for predicting biodiversity change and supporting sound management actions to conservation and sustainable use of natural resources (Zwiener et al., 2017).

Studies on how plant species and communities respond to environmental gradients have applied different univariate and multivariate analytical methods and techniques (Anderson et al., 2011). Among multivariate methods, canonical analyses that directly compare species composition and a set of environmental descriptors are important tools to evaluate hypotheses formulated by community ecology (Anderson et al., 2011; Legendre \& Legendre, 2012). This type of analysis can be used to detect relationships between species and the environment and investigate specific issues of occurrence and responses to the variables considered.
Given the importance of understanding how communities are structured and the scarcity of information on how local environmental variables interact with the MOF vegetation, our study aimed to assess plant community structure and the influence of different edaphic variables on tree biodiversity. More specifically, we were interested in: (i) describing community structure of trees, pedological units where they occur and variation in soil attributes; (ii) assessing and identifying tree species that characterize communities over different soil conditions; and (iii) investigating correlations of chemical and physical soil properties with community composition across a pedological gradient using multivariate analyses.

\section{MATERIALS AND METHODS}

\subsection{Study sites}

This study was conducted in a forest fragment at the "Três Capões" farm $\left(25^{\circ} 25^{\prime} 18^{\prime \prime} \mathrm{S}\right.$ and $\left.51^{\circ} 41^{\prime} 45^{\prime \prime} \mathrm{W}\right)$ within the municipality of Guarapuava, state of Paraná, Southern Brazil (Figure 1). According to long-standing residents and staff, the forest in the property has never been clear-cut; however, some areas may have suffered selective logging of species with economic value.

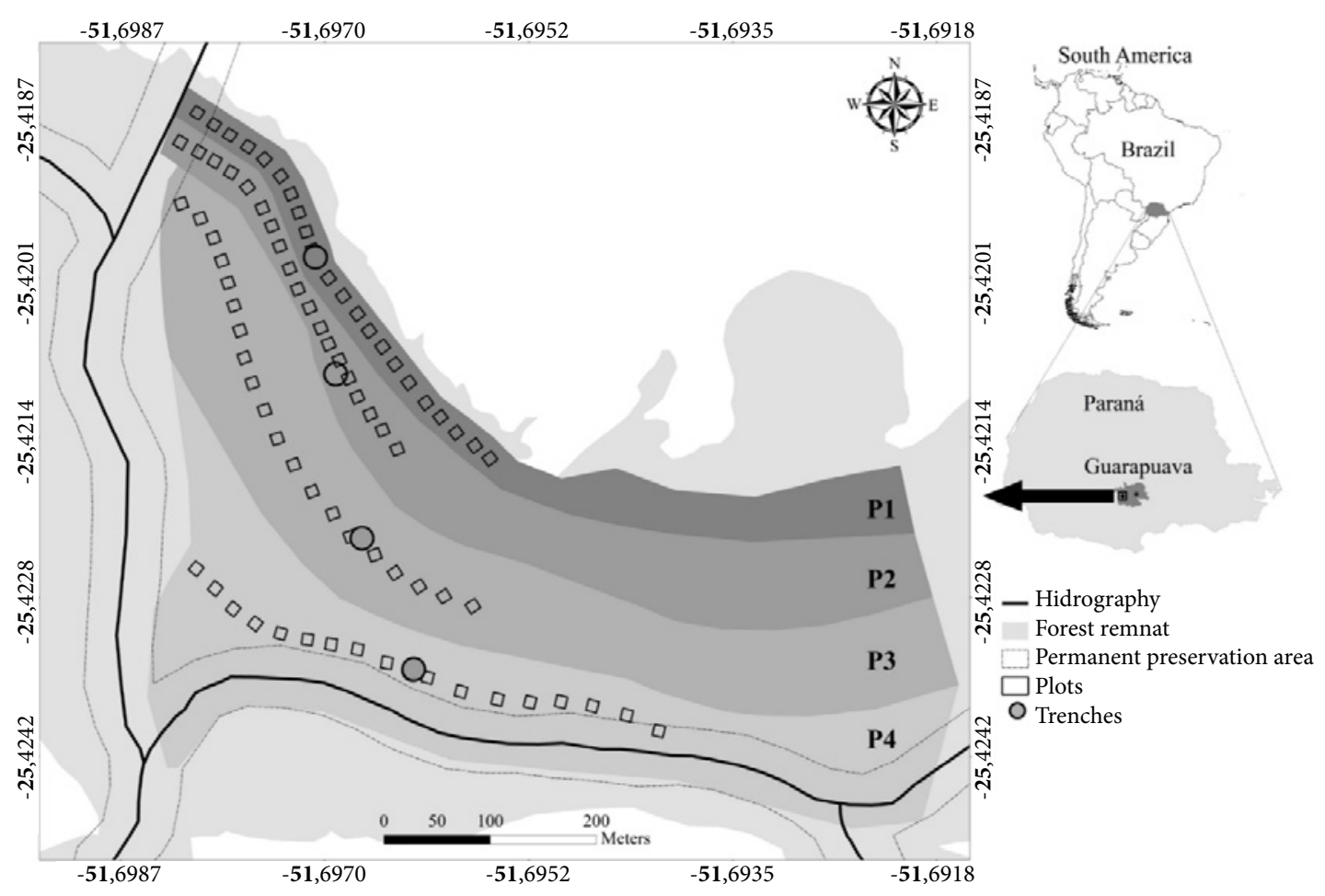

Figure 1. Sampled sites in "Três Capões" farm, Guarapuava, Southern Brazil. The trenches where the soil was characterized and sampled are represented by filled gray circles.

Source: Munhoz (2018). 
The region is located in the Paraná Sedimentary Basin, with extrusive igneous rocks of the Serra Geral Formation (Mineropar, 2001). Smooth to undulate relief with convexconcave ramps of small declive are predominant and the altimetric gradient varies from $960 \mathrm{~m}$ to $935 \mathrm{~m}$ (Mineropar, 2006). The vegetation is represented by the montane Mixed Ombrophilous Forest. According to Köppen's classification, the climate is Cfb, without dry season (Peel et al., 2007). Average temperature in the winter is $12.8^{\circ} \mathrm{C}$ and in the summer, $23.5^{\circ} \mathrm{C}$. Average annual rainfall is $1,944 \mathrm{~mm}$.

\subsection{Soil and vegetation sampling}

Pedological unit descriptions were based on four trenches distributed in the forest fragment (Figure 1). Soil samples were analyzed at the Laboratory of the Universidade Federal do Paraná. Soil units were classified according to the Brazilian Soil Classification System (Embrapa, 2013). Soil drainage classification was based on Curcio et al. (2006) as follows: markedly drained, well and moderately drained to non-hydromorphic soils and imperfectly drained to semihydromorphic soils. To include these classes in the analyses we assigned ascending ordinal values to the drainage classes, according to increasing degree of hydromorphy: (1) markedly drained; (2) well drained; (3) seasonal moderately drained; and (4) imperfectly drained.

The forest fragment was divided into four sites with different soil characteristics (P1, P2, P3, and P4) based on the pedological classification and geomorphic form. In each site, we sampled the vegetation with fixed area plots of $10 \times 10 \mathrm{~m}$, placed on a transect in the E-W direction, with a $2 \mathrm{~m}$ interval between each plot (Figure 1). We sampled a total of 80 plots $(0.8 \mathrm{ha})$ in all sites, of which 22 plots were sampled on P1 (0.22 ha), 20 plots on P2 (0.20 ha), 22 plots on P3 (0.22 ha) and 16 plots on P4 (0.16 ha). All sites reached sampling adequacy according to the cumulative mean number of species in the plots (Mueller-Dombois \& Ellenberg, 1974) (Figure 2), considering that the accumulated values were within $2.5 \%$ (above or below) deviations of the mean (Rodal et al., 2013).
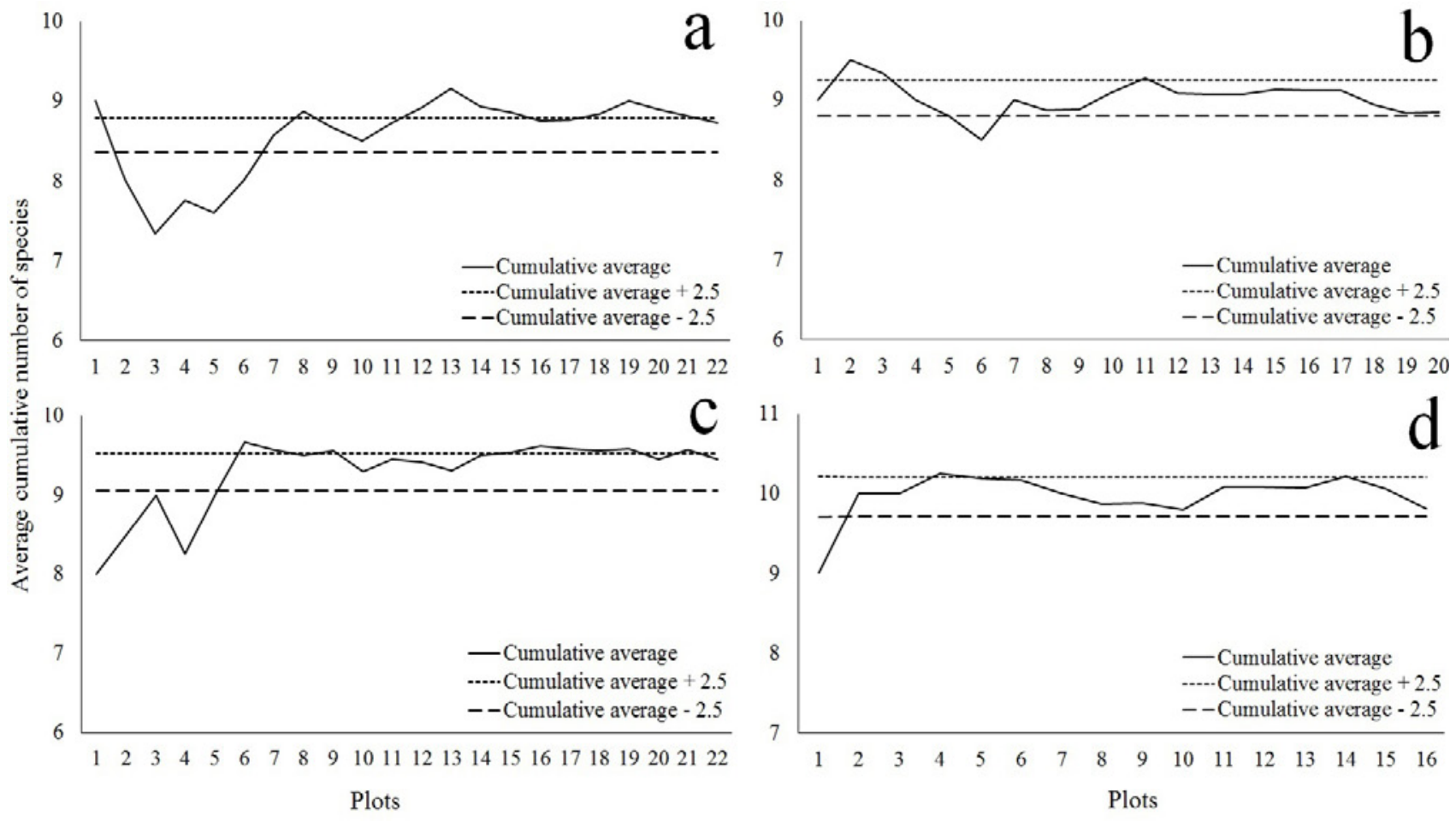

Figure 2. Average cumulated number species in the four sites - (a) P1, (b) P2, (c) P3, and (d) P4 - of the remnant of the Mixed Forest of the Três Capões Farm. 
All arboreal individuals with circumference at breast height $\geq 15 \mathrm{~cm}$ (equivalent to $\mathrm{DBH} \geq 4.8 \mathrm{~cm}$ ) were included in the sample and identified. Dead standing trees were grouped into a separated category. The nomenclature of families and genera were based on the APG IV system and the species were verified in the archives of Flora do Brasil (JBRJ, [2018?]).

\subsection{Data analysis}

Tree communities were described using phytosociological analysis (density, frequency, dominance and importance value). The indicator value index (IndVal) was used to identify typical species of different soil conditions (sites P1, P2, P3, and P4) across the environmental gradient (Dufrêne \& Legendre, 1997). The indicator value of a species is given by the product of its site specificity (Aij) and its site fidelity $(B i j): \operatorname{IndVal} i j=A i j \times B i j \times 100$, where $A i j$ is the abundance of species $i$ across plots in site $j$ divided by the abundance of species $i$ over all sites, and $B i j$ is the number of plots in site $j$ where species $i$ is present, divided by the total number of plots in site $j$. IndVal is maximized (100\%) when all individuals of species $i$ are found in one site and when that species occurs in all of the plots in that site. Statistical significance of the IndVal of each species was evaluated with 999 permutations of plots among the four sites (Dufrêne \& Legendre, 1997).

We used Redundancy Analysis (RDA), which is a form of canonical multivariate ordination, to assess the relative importance of edaphic variables on structuring composition patterns of species in the communities (Legendre \& Legendre, 2012). The matrix of environmental variables was initially constructed considering all variables. To avoid misleading interpretation of RDA results, we selected the least correlated or collinear variables after performing a principal component analysis (PCA) based on a correlation matrix of standardized variables (Figure 3 ).

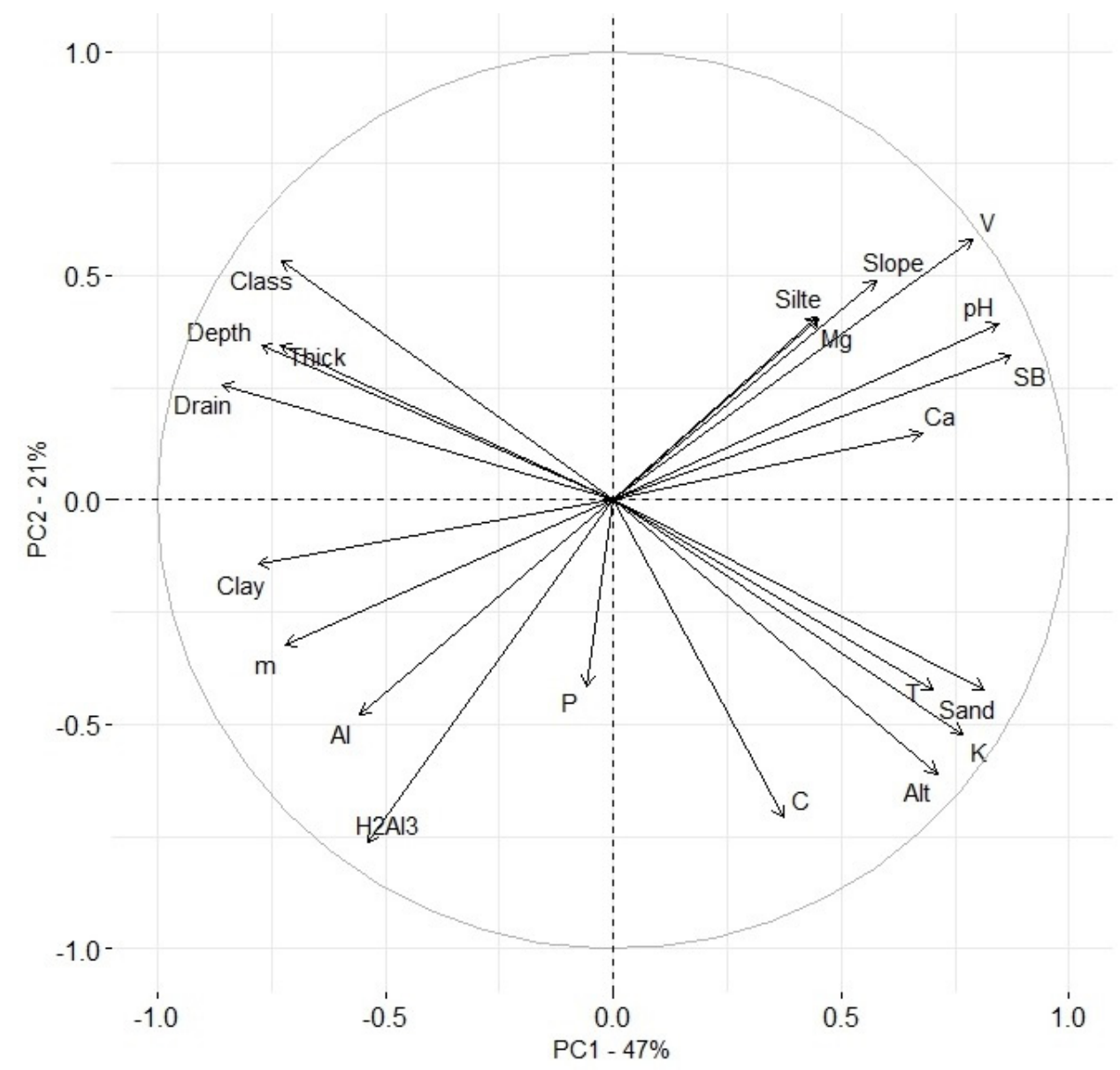

Figure 3. Loadings plot of principal component analysis with soil variables (see methods for further description of variables). 
The selected variables for the RDA model were: $\mathrm{H}+\mathrm{Al}^{+3}$ $\left(\mathrm{HAl}^{3}\right), \mathrm{pH}$ in $\mathrm{CaCl}_{2}(\mathrm{pH})$, Aluminum (Al), Phosphorus (P), Cation exchange capacity at $\mathrm{pH} 7.0(\mathrm{~T})$, Base saturation percentage (V), Organic Carbon (C), Clay, Soil Type (Class) and Drainage (Drain). We performed the Monte Carlo test to evaluate the statistical significance of the full RDA model, individual axes and environmental variables (Ter Braak \& Prentice, 2004). Significance was assessed by comparing empirical values with 999 unrestricted permutations, considering significant results when $p<0.05$. All statistical analyses were performed in the $\mathrm{R}$ environment ( $\mathrm{R}$ Core Team, 2016).

\section{RESULTS}

We sampled 1,617 individuals from 55 species, with a 2,021 ind ha $^{-1}$ overall density and a $58.89 \mathrm{~m}^{2} \mathrm{ha}^{-1}$ basal area. The species richness, density, and basal area were not evenly distributed across sampling sites. Site P1 had 37 species, with a 2431 ind ha ${ }^{-1}$ density and a $48.44 \mathrm{~m}^{2}$ ha-1 basal area. Site P2 had 35 species, with a 1,695 ind ha-1 density and a $64.23 \mathrm{~m}^{2} \mathrm{ha}^{-1}$ basal area. Site P3 was had 35 species, with a 1923 ind ha ${ }^{-1}$ density and a $64.26 \mathrm{~m}^{2} \mathrm{ha}^{-1}$ basal area, whereas $\mathrm{P} 4$ had 32 species, with a 2,000 ind ha ${ }^{-1}$ density and a $59.6 \mathrm{~m}^{2} \mathrm{ha}^{-1}$ basal area. Phytosociological results of tree communities in each site are shown in Table 1.

The indicator value index (IndVal) estimated for species in the four sites showed species that have significant distinct responses to the edaphic gradient and characterize different soil conditions (Table 2). These results were closely followed and corroborated by phytosociological descriptors of the horizontal structure of vegetation (Table 1). The predilection of species was evidenced by the IndVal and importance (IV) values obtained by the main species that form the forest horizontal structure in each site. P1: Lithraea molleoides (IndVal: 0.66; Ranking IV: 2), Gymnanthes klotzschiana (IndVal: 0.45; Ranking IV: 1); P2: Muellera campestris (IndVal: 0.39; Ranking IV: 1); P3: Araucaria angustifolia (IndVal: 0.46; Ranking IV: 1), Campomanesia xanthocarpa (IndVal: 0.38; Ranking IV: 3), Eugenia uniflora (IndVal: 0.37; Ranking IV: 2); P4: Nectandra megapotamica (IndVal: 0.47; Ranking IV: 2). These species, although present in almost all sites, showed greater specificity and affinity to distinct environmental conditions.

Table 1. Phytosociological variables of tree species with the highest importance values in the MOF remnant of "Três Capões" farm, Guarapuava, Southern Brazil.

\begin{tabular}{|c|c|c|c|c|c|c|c|c|c|}
\hline Site & Species & NI & $\begin{array}{c}\mathrm{AD} \\
\text { ind/ha }\end{array}$ & $\begin{array}{l}\text { ADo } \\
\mathbf{m}^{2} / \mathrm{ha}\end{array}$ & $\begin{array}{c}\text { AF } \\
\%\end{array}$ & $\begin{array}{c}\text { RD } \\
\%\end{array}$ & $\begin{array}{c}\text { RDo } \\
\%\end{array}$ & $\begin{array}{c}\text { RF } \\
\%\end{array}$ & $\begin{array}{l}\text { IV } \\
\%\end{array}$ \\
\hline \multirow{15}{*}{$\vec{a}$} & Gymnanthes klotzschiana Müll.Arg. & 156 & 709.09 & 10.51 & 77.27 & 29.16 & 21.71 & 8.90 & 19.92 \\
\hline & Lithraea molleoides (Vell.) Engl. & 42 & 190.91 & 9.98 & 68.18 & 7.85 & 20.61 & 7.85 & 12.10 \\
\hline & Eugenia uniflora L. & 41 & 186.36 & 1.86 & 72.73 & 7.66 & 3.85 & 8.38 & 6.63 \\
\hline & $\begin{array}{l}\text { Muellera campestris (Mart. ex Benth.) } \\
\text { M.J. Silva e A.M.G. Azevedo }\end{array}$ & 22 & 100.00 & 5.39 & 22.73 & 4.11 & 11.13 & 2.62 & 5.95 \\
\hline & Cinnamodendron dinisii Schwacke & 29 & 131.82 & 2.28 & 59.09 & 5.42 & 4.72 & 6.81 & 5.65 \\
\hline & Guettarda uruguensis Cham. e Schltdl. & 42 & 190.91 & 1.37 & 40.91 & 7.85 & 2.82 & 4.71 & 5.13 \\
\hline & Styrax leprosus Hook. et Arn. & 31 & 140.91 & 2.28 & 36.36 & 5.79 & 4.70 & 4.19 & 4.89 \\
\hline & $\begin{array}{l}\text { Allophylus edulis (A.St.-Hil. et al.) Hieron. ex } \\
\text { Niederl }\end{array}$ & 23 & 104.55 & 0.76 & 54.55 & 4.30 & 1.57 & 6.28 & 4.05 \\
\hline & Machaerium paraguariense Hassl. & 9 & 40.91 & 3.23 & 22.73 & 1.68 & 6.67 & 2.62 & 3.66 \\
\hline & Eugenia pyriformis Cambess. & 11 & 50.00 & 1.82 & 27.27 & 2.06 & 3.76 & 3.14 & 2.99 \\
\hline & Erythroxylum deciduum A.St.-Hil. & 8 & 36.36 & 1.89 & 27.27 & 1.50 & 3.91 & 3.14 & 2.85 \\
\hline & Dalbergia frutescens (Vell.) Britton & 16 & 72.73 & 0.52 & 27.27 & 2.99 & 1.07 & 3.14 & 2.40 \\
\hline & Zanthoxylum rhoifolium Lam. & 10 & 45.45 & 0.32 & 27.27 & 1.87 & 0.67 & 3.14 & 1.89 \\
\hline & Zanthoxylum petiolare A.St.-Hil. \& Tul. & 7 & 31.82 & 0.16 & 27.27 & 1.31 & 0.33 & 3.14 & 1.59 \\
\hline & Campomanesia xanthocarpa (Mart.) O.Berg & 7 & 31.82 & 0.28 & 18.18 & 1.31 & 0.58 & 2.09 & 1.33 \\
\hline
\end{tabular}


Table 1. Continued...

\begin{tabular}{|c|c|c|c|c|c|c|c|c|c|}
\hline Site & Species & NI & $\begin{array}{l}\text { AD } \\
\text { ind/ha }\end{array}$ & $\begin{array}{l}\text { ADo } \\
\mathbf{m}^{2} / \mathrm{ha}\end{array}$ & $\begin{array}{c}\mathrm{AF} \\
\%\end{array}$ & $\begin{array}{c}\text { RD } \\
\%\end{array}$ & $\begin{array}{c}\text { RDo } \\
\%\end{array}$ & $\begin{array}{c}\text { RF } \\
\%\end{array}$ & $\begin{array}{l}\text { IV } \\
\%\end{array}$ \\
\hline \multirow{15}{*}{$\tilde{n}$} & $\begin{array}{l}\text { Muellera campestris (Mart. ex Benth.) M.J. Silva e } \\
\text { A.M.G. Azevedo }\end{array}$ & 42 & 210.00 & 8.52 & 75.00 & 12.39 & 13.26 & 8.62 & 11.40 \\
\hline & Eugenia uniflora L. & 55 & 275.00 & 3.45 & 70.00 & 16.22 & 5.36 & 8.05 & 9.90 \\
\hline & Nectandra megapotamica (Spreng.) Mez & 8 & 40.00 & 13.38 & 25.00 & 2.36 & 20.83 & 2.87 & 8.70 \\
\hline & Araucaria angustifolia (Bertol.) Kuntze & 6 & 30.00 & 11.16 & 30.00 & 1.77 & 17.38 & 3.45 & 7.50 \\
\hline & Myrcianthes pungens (O. Berg) D. Legrand & 27 & 135.00 & 2.84 & 50.00 & 7.96 & 4.42 & 5.75 & 6.00 \\
\hline & Campomanesia xanthocarpa (Mart.) O.Berg & 22 & 110 & 2.75 & 50.00 & 6.49 & 4.28 & 5.75 & 5.50 \\
\hline & Castela tweedii Planch. & 27 & 135 & 0.45 & 65.00 & 7.96 & 0.70 & 7.47 & 5.40 \\
\hline & Celtis iguanaea (Jacq.) Sarg. & 20 & 100 & 0.80 & 50.00 & 5.90 & 1.25 & 5.75 & 4.30 \\
\hline & Gymnanthes klotzschiana Müll.Arg. & 15 & 75 & 2.71 & 35.00 & 4.42 & 4.21 & 4.02 & 4.20 \\
\hline & Styrax leprosus Hook. et Arn. & 12 & 60 & 1.34 & 30.00 & 3.54 & 2.09 & 3.45 & 3.00 \\
\hline & Casearia decandra Jacq. & 14 & 70 & 0.81 & 30.00 & 4.13 & 1.26 & 3.45 & 2.90 \\
\hline & Eugenia pyriformis Cambess. & 8 & 40 & 1.55 & 30.00 & 2.36 & 2.42 & 3.45 & 2.70 \\
\hline & Machaerium paraguariense Hassl. & 10 & 50 & 1.29 & 20.00 & 2.95 & 2.01 & 2.30 & 2.40 \\
\hline & Matayba elaeagnoides Radlk. & 2 & 10 & 2.98 & 10.00 & 0.59 & 4.64 & 1.15 & 2.10 \\
\hline & Strychnos pseudoquina A.St.-Hil. & 6 & 30 & 0.31 & 30.00 & 1.77 & 0.49 & 3.45 & 1.90 \\
\hline \multirow{15}{*}{$\hat{n}$} & Araucaria angustifolia (Bertol.) Kuntze & 25 & 113.64 & 26.25 & 77.27 & 5.91 & 40.84 & 8.13 & 18.29 \\
\hline & Eugenia uniflora $L$. & 94 & 427.27 & 4.27 & 95.45 & 22.22 & 6.64 & 10.05 & 12.97 \\
\hline & Campomanesia xanthocarpa (Mart.) O. Berg & 50 & 227.27 & 4.00 & 86.36 & 11.82 & 6.23 & 9.09 & 9.05 \\
\hline & Nectandra megapotamica (Spreng.) Mez & 21 & 95.45 & 8.43 & 59.09 & 4.96 & 13.11 & 6.22 & 8.10 \\
\hline & Banara tomentosa Clos. & 41 & 186.36 & 1.36 & 63.64 & 9.69 & 2.12 & 6.70 & 6.17 \\
\hline & Brunfelsia pilosa Plowman & 35 & 159.09 & 0.42 & 72.73 & 8.27 & 0.65 & 7.66 & 5.53 \\
\hline & $\begin{array}{l}\text { Muellera campestris (Mart. ex Benth.) M.J. Silva e } \\
\text { A.M.G. Azevedo }\end{array}$ & 15 & 68.18 & 5.38 & 31.82 & 3.55 & 8.36 & 3.35 & 5.09 \\
\hline & Styrax leprosus Hook. et Arn. & 17 & 77.27 & 1.62 & 50.00 & 4.02 & 2.52 & 5.26 & 3.93 \\
\hline & Casearia decandra Jacq. & 15 & 68.18 & 1.61 & 31.82 & 3.55 & 2.51 & 3.35 & 3.13 \\
\hline & Eugenia pyriformis Cambess. & 8 & 36.36 & 0.60 & 31.82 & 1.89 & 0.93 & 3.35 & 2.06 \\
\hline & Campomanesia guazumifolia (Cambess.) O.Berg & 7 & 31.82 & 0.63 & 31.82 & 1.65 & 0.98 & 3.35 & 1.99 \\
\hline & Vitex megapotamica (Spreng.) Moldenke & 5 & 22.73 & 2.14 & 13.64 & 1.18 & 3.33 & 1.44 & 1.98 \\
\hline & Celtis iguanaea (Jacq.) Sarg. & 9 & 40.91 & 0.36 & 27.27 & 2.13 & 0.56 & 2.87 & 1.85 \\
\hline & Gymnanthes klotzschiana Müll.Arg. & 9 & 40.91 & 0.32 & 22.73 & 2.13 & 0.50 & 2.39 & 1.67 \\
\hline & Cupania vernalis Cambess. & 2 & 9.09 & 2.02 & 9.09 & 0.47 & 3.15 & 0.96 & 1.53 \\
\hline \multirow{15}{*}{$\vec{a}$} & Gymnanthes klotzschiana Müll.Arg. & 62 & 387.50 & 18.68 & 87.50 & 19.40 & 31.30 & 9.00 & 19.90 \\
\hline & Nectandra megapotamica (Spreng.) Mez & 47 & 293.80 & 8.46 & 68.80 & 14.70 & 14.20 & 7.10 & 12.00 \\
\hline & Campomanesia xanthocarpa (Mart.) O. Berg & 25 & 156.30 & 7.75 & 81.30 & 7.80 & 13.00 & 8.40 & 9.70 \\
\hline & Eugenia uniflora L. & 31 & 193.80 & 1.48 & 75.00 & 9.70 & 2.50 & 7.70 & 6.60 \\
\hline & Styrax leprosus Hook. et Arn. & 20 & 125.00 & 2.16 & 75.00 & 6.30 & 3.60 & 7.70 & 5.90 \\
\hline & Machaerium paraguariense Hassl. & 13 & 81.30 & 3.53 & 43.80 & 4.10 & 5.90 & 4.50 & 4.80 \\
\hline & Araucaria angustifolia (Bertol.) Kuntze & 6 & 37.50 & 2.98 & 37.50 & 1.90 & 5.00 & 3.90 & 3.60 \\
\hline & Brunfelsia pilosa Plowman & 17 & 106.30 & 0.27 & 43.80 & 5.30 & 0.50 & 4.50 & 3.40 \\
\hline & Banara tomentosa Clos & 13 & 81.30 & 0.50 & 50.00 & 4.10 & 0.80 & 5.20 & 3.40 \\
\hline & Strychnos brasiliensis (Spreng.) Mart. & 15 & 93.80 & 0.86 & 37.50 & 4.70 & 1.40 & 3.90 & 3.30 \\
\hline & $\begin{array}{l}\text { Muellera campestris (Mart. ex Benth.) M.J. Silva } \\
\text { \& A.M.G. Azevedo }\end{array}$ & 7 & 43.80 & 2.30 & 37.50 & 2.20 & 3.90 & 3.90 & 3.30 \\
\hline & Casearia decandra Jacq. & 9 & 56.30 & 0.57 & 43.80 & 2.80 & 0.90 & 4.50 & 2.80 \\
\hline & $\begin{array}{l}\text { Allophylus edulis (A.St.-Hil. et al.) Hieron. ex } \\
\text { Niederl }\end{array}$ & 9 & 56.30 & 0.33 & 43.80 & 2.80 & 0.60 & 4.50 & 2.60 \\
\hline & Campomanesia guazumifolia (Cambess.) O. Berg & 10 & 62.50 & 0.66 & 31.30 & 3.10 & 1.10 & 3.20 & 2.50 \\
\hline & Prunus myrtifolia (L.) Urb. & 3 & 18.80 & 2.23 & 18.80 & 0.90 & 3.70 & 1.90 & 2.20 \\
\hline
\end{tabular}

MOF: Mixed Ombrophilous Forest; NI: number of individuals; AD: absolute density; ADo: absolute dominance; AF: absolute frequency; RD: relative density; RDo: relative dominance; RF: relative frequency; IV\%: relative importance value. 
Table 2. Indicator species in decreasing order of the index value (IndVal) and ranking of importance (IV \%) in the four sites (P1, P2, P3 and P4). The significance of IndVal values was based on 999 permutations.

\begin{tabular}{|c|c|c|c|}
\hline Species & IndVal & $p$ & $\begin{array}{c}\text { Ranking } \\
\text { IV \% }\end{array}$ \\
\hline \multicolumn{4}{|l|}{ P1 } \\
\hline Lithraea molleoides & 0.66 & 0.001 & 2 \\
\hline Cinnamodendron dinisii & 0.45 & 0.001 & 5 \\
\hline Gymnanthes klotzschiana & 0.45 & 0.001 & 1 \\
\hline Guettarda uruguensis & 0.40 & 0.001 & 6 \\
\hline Allophylus edulis & 0.29 & 0.010 & 8 \\
\hline \multicolumn{4}{|l|}{ P2 } \\
\hline Castela tweedii & 0.44 & 0.001 & 7 \\
\hline Celtis iguanaea & 0.44 & 0.001 & 8 \\
\hline Muellera campestris & 0.39 & 0.002 & 1 \\
\hline Myrcianthes pungens & 0.32 & 0.003 & 5 \\
\hline Cestrum intermedium & 0.14 & 0.048 & 25 \\
\hline \multicolumn{4}{|l|}{$\mathrm{P} 3$} \\
\hline Araucaria angustifolia & 0.46 & 0.001 & 1 \\
\hline Banara tomentosa & 0.42 & 0.001 & 5 \\
\hline Campomanesia xanthocarpa & 0.38 & 0.002 & 3 \\
\hline Eugenia uniflora & 0.37 & 0.002 & 2 \\
\hline Brunfelsia pilosa & 0.35 & 0.002 & 6 \\
\hline \multicolumn{4}{|l|}{ P4 } \\
\hline Nectandra megapotamica & 0.47 & 0.001 & 2 \\
\hline Machaerium paraguariense & 0.29 & 0.004 & 6 \\
\hline Strychnos brasiliensis & 0.25 & 0.011 & 10 \\
\hline
\end{tabular}

Results of the RDA (Table 3) generated eigenvalues of 1.20 (axis 1), 0.48 (axis 2) and 0.36 (axis 3). The overall variance of species composition in all sites, represented by the first two axes combined, was $26 \%$, whereas the variance in species composition, represented by the axes in relation to all variance explained by soil variables, reached $62 \%$.

Table 3. Eigenvalues. Variance explained and loadings of the first three axes of the constrained RDA ordination. The RDA was performed with tree species composition and soil variables across in the four sites. Cumulative proportion corresponds the overall variance represented by the axes combined, whereas constrained proportion corresponds to the variance represented by the axes in relation to all variance explained by soil variables.

\begin{tabular}{lccc|} 
& RDA1 & RDA2 & RDA3 \\
Eigenvalue & 1.20 & 0.48 & 0.36 \\
Proportion explained & 0.18 & 0.07 & 0.05 \\
Cumulative proportion & 0.18 & 0.26 & 0.31 \\
Constrained proportion & 0.44 & 0.62 & 0.75
\end{tabular}

Table 3. Continued...

\begin{tabular}{lccc}
$\begin{array}{l}\text { Loadings of soil } \\
\text { variables }\end{array}$ & RDA1 & RDA2 & RDA3 \\
\hline $\mathrm{pH}$ & -0.06 & $\mathbf{0 . 6 9}$ & -0.16 \\
\hline $\mathrm{Al}$ & 0.23 & -0.39 & 0.22 \\
\hline $\mathrm{T}$ & 0.46 & 0.43 & 0.03 \\
\hline $\mathrm{P}$ & 0.08 & -0.16 & 0.16 \\
\hline $\mathrm{HAl}^{3}$ & $\mathbf{0 . 5 4}$ & $\mathbf{- 0 . 5 6}$ & 0.29 \\
\hline $\mathrm{C}$ & $\mathbf{0 . 6 2}$ & 0.24 & 0.12 \\
\hline $\mathrm{V}$ & -0.28 & $\mathbf{0 . 7 1}$ & -0.17 \\
\hline Clay & -0.10 & $\mathbf{- 0 . 7 4}$ & 0.40 \\
\hline Drain & -0.36 & $\mathbf{- 0 . 7 9}$ & -0.31 \\
\hline Class & $\mathbf{- 0 . 6 0}$ & $\mathbf{- 0 . 6 0}$ & -0.38 \\
\hline
\end{tabular}

RDA: redundancy analysis; $\mathrm{T}$ : cation exchange capacity at $\mathrm{pH}$ 7.0; C: organic carbon; V: base saturation percentage.

The permutation tests confirmed that the results generated by the RDA differ significantly from random results. Both the general model $(\mathrm{F}=4.8853, p=0.001)$ and the first six axes and environmental variables have a greater explanation than random data (Table 4).

Table 4. Permutation test of the full model (RDA global), individual RDA axes (RDA1-RDA6) and soil variables to compare empirical to random ordination values. Significance was assessed with 999 permutations.

\begin{tabular}{lcccc}
\multicolumn{1}{c}{ Model } & Df & Variance & F & $p$ \\
\hline RDA global & 10 & 2.7245 & 4.88 & 0.001 \\
RDA1 & 1 & 1.2028 & 21.5676 & 0.001 \\
\hline RDA2 & 1 & 0.4847 & 8.6903 & 0.001 \\
\hline RDA3 & 1 & 0.3622 & 6.4942 & 0.001 \\
\hline RDA4 & 1 & 0.2582 & 4.6288 & 0.001 \\
\hline RDA5 & 1 & 0.1676 & 3.0043 & 0.001 \\
\hline RDA6 & 1 & 0.0945 & 1.6940 & 0.038 \\
pH & 1 & 0.3128 & 5.6083 & 0.001 \\
\hline Al & 1 & 0.2223 & 3.9864 & 0.002 \\
\hline T & 1 & 0.4477 & 8.0275 & 0.001 \\
\hline P & 1 & 0.1457 & 2.6129 & 0.012 \\
\hline HAl & 1 & 0.5880 & 10.5434 & 0.001 \\
\hline C & 1 & 0.1275 & 2.2861 & 0.015 \\
\hline V & 1 & 0.2859 & 5.1261 & 0.001 \\
\hline Clay & 1 & 0.2890 & 5.1816 & 0.001 \\
\hline Drain & 1 & 0.1999 & 3.5839 & 0.002 \\
\hline Class & 1 & 0.1058 & 1.8964 & 0.045 \\
\hline
\end{tabular}

RDA: redundancy analysis; Df: degrees of freedom; $F$ : $F$ value; $p$ : $p$-value; T: cation exchange capacity at $\mathrm{pH}$ 7.0; C: organic carbon; V: base saturation percentage. 
Edaphic variables that distinguish the sites and pedological units, such as water regime and chemical and physical characteristics of soils, influenced the floristic composition and vegetation structure. The sites formed relatively well-defined groups in the RDA biplot that reflected distinct responses to the edaphic gradient. The sites $\mathrm{P} 3$ and $\mathrm{P} 4$ were more closely grouped according to floristic similarity influenced by soil characteristics, whereas the vegetation in sites $\mathrm{P} 1$ and $\mathrm{P} 2$ showed more distinct responses to the edaphic gradient (Figure 4a).

The plots on site P1 are located on Haplic Cambisol, where the major influences are higher levels of $\mathrm{H}^{+\mathrm{Al}^{+3}}$ and $\mathrm{C}$, which results in exchangeable acidity and are also subject to seasonal water influence. For the site P2, natural fertility was the variable that mostly correlated with community composition, represented by higher $\mathrm{pH}$ values of Regolitic Neosols and base saturation percentage (V). The plots on P3 were influenced by the greater thickness of Latosol Bruno, the absence of hydromorphy (higher drainage) and lower $\mathrm{pH}$. In $\mathrm{P} 4$, the position of plots indicated an influence of variables related to Humic Cambisol, lower $\mathrm{pH}$ and poor drainage (imperfectly drained).

The RDA results also revealed the contribution of species to the distribution and ordering of plots related to species' responses to the edaphic gradient (Figure 4b). Cinnamodendron dinisii, Guettarda uruguensis, Gymnanthes klotzschiana, and Lithraea molleoides responded to environments with higher carbon contents as recorded in P1 occurring on Haplic Cambisol and in the case of G. klotzschiana to lower $\mathrm{pH}$ and higher concentrations of $\mathrm{H}^{+}+\mathrm{Al}^{+3}$. Celtis iguanaea, Castella tweedii, Muellera campestris and Myrcianthes pungens influenced the ordering of site P2 occurring on Regolithic Neosol that showed the highest $\mathrm{pH}$ index and base saturation percentage $(V)$ when compared with the other areas. Plots on site $\mathrm{P} 3$ were grouped according to responses of Araucaria angustifolia, Banara tomentosa, Brunfelsia pilosa, Campomanesia xanthocarpa and Eugenia uniflora to well-drained soils with a higher clay concentration, whereas site $\mathrm{P} 4$ was influenced by Nectandra megapotamica and species with generalist behavior towards soil attributes, such as Annona cacans, Campomanesia guazumifolia, Casearia decandra, Dalbergia frutescens, Eugenia pyriformis, Machaerium paraguariense, Strychnos brasiliensis, Styrax leprosus, Vitex megapotamica, and Zanthoxylum rhoifolium. These species were found with similar abundance in more than one site.

Some species did not influence the ordination of sites as result of scores generated from the RDA that were distinct when compared with other species. For example, Allophylus edulis was common in sites with high $\mathrm{Al}^{+3}$ concentration, whereas Nectandra megapotamica prefers places with greater clay and soil moisture concentrations and Gymnanthes klotzschiana was strongly influenced by lower $\mathrm{pH}$ due to the higher concentrations of $\mathrm{H}+\mathrm{Al}^{+3}$.
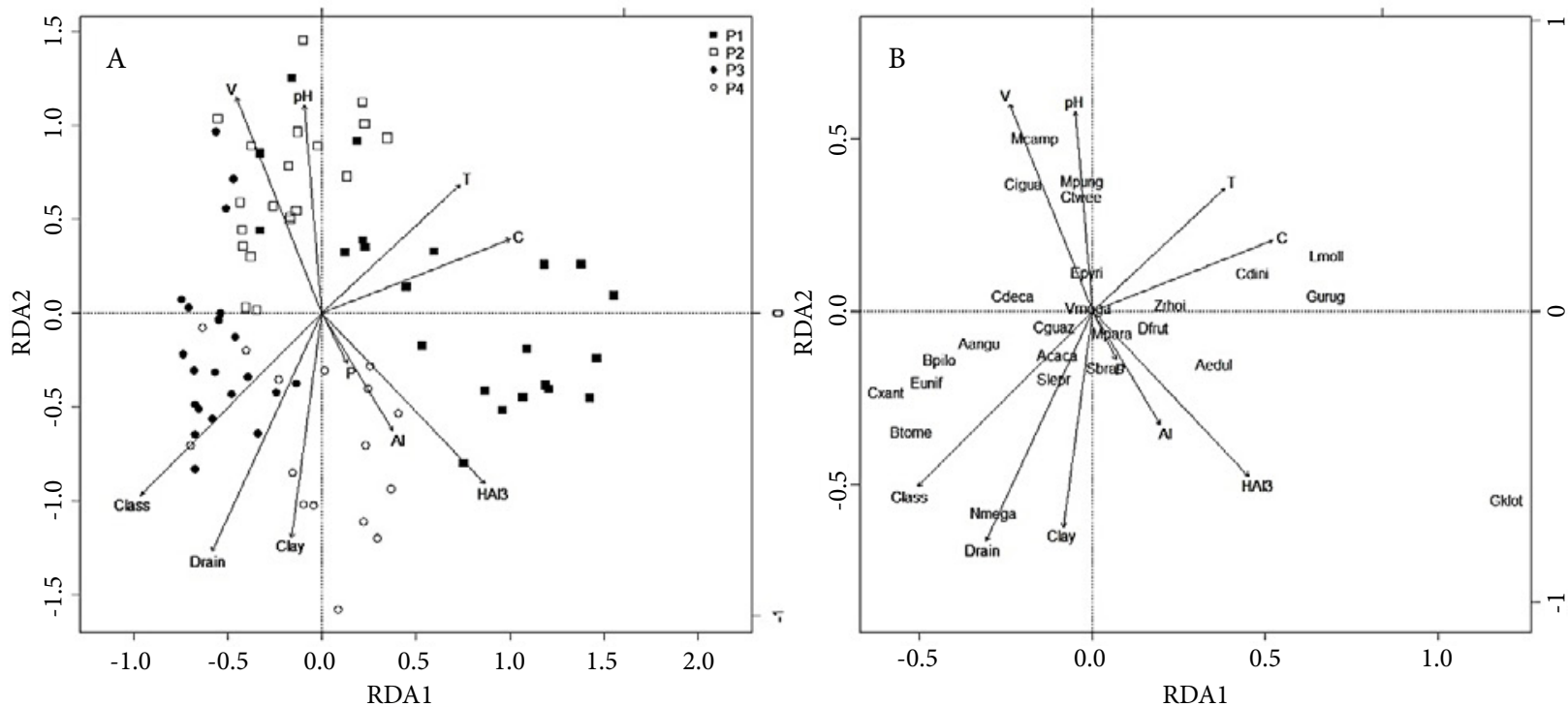

Figure 4. Redundancy analysis biplot showing ordination of the sampling units (a) over Haplic Cambisol (P1), Regolithic Neosol (P2), Latosol Bruno (P3) and Humic Cambisol (P4), and species with 10 or + individuals (b).

Gklotz: Gymnanthes klotzschiana; Eunif: Eugenia uniflora; Aangu: Araucaria angustifolia; Mcamp: Muellera campestris; Cxanth: Campomanesia xanthocarpa; Nmega: Nectandra megapotamica; Slepr: Styrax leprosus; Lmoll: Lithrea molleoides; Btome: Banara tomentosa; Bpilo: Brunfelsia pilosa; Mpara: Machaerium paraguariense; Cdeca: Casearia decandra; Cdini: Cinnamodendron dinisii; Epyri: Eugenia pyriformis; Mpung: Myrcianthes pungens; Adeul: Allophylus edulis; Cguaz: Campomanesia guazumifolia; Cigua: Celtis iguanaea; Gurug: Guettarda uruguensis; Ctwee: Castela tweedii; Sbras: Strychnos brasiliensis; Vmega: Vitex megapotamica; Dfrut: Dalbergia frutescens; Acaca: Annona cacans; Zrhoi: Zanthoxylum rhoifolium). Soil variables are represented by codes (see methods). 


\section{DISCUSSION}

Species in plant communities have different behaviors in relation to environmental factors (Hirst \& Jackson, 2007). The use of ordination techniques allows detecting the relationships of species with the environment and how their responses affect patterns of biodiversity. RDA ordination demonstrates species-environment associations for different classes of soils. Such results complement and corroborate phytosociological and indicator species results, and contribute to the understanding of how communities are structured and assembled in the MOF.

Forest habitat classification according to soil characteristics represents an important starting point to understand local differences in the floristic composition of MOF. Soil attributes are responsible for the genesis of resource availability for plants such as water and nutrients, which, among other factors, regulate plant species occurrences and abundance and may be considered an important element for the segregation of habitats (Resende et al., 2002). It was evident that the soil shows variations at small distances and its characteristics correlate with vegetation patterns.

The horizontal structure of the forest in each site showed a set of main species that influences tree community structure; however, these species were not exclusive to communities of a given soil type. In the case of the P1, Gymnanthes klotzschiana was the species that stood out because it obtained the highest values in all phytosociological variables, presented a significand indicator value and influenced in the RDA results. This predominance of G. klotzschiana in soils with higher moisture was also reported by Barddal et al. (2004), Curcio et al. (2007) and Silva et al. (2010).

Muellera campestris, Eugenia uniflora, Nectandra megapotamica, and Myrcianthes pungens were the most representative species in plots within site P2. These species also occur in other phytophysiognomy of the Atlantic Forest (JBRJ, [2018?]). Interestingly, none of them has been previously described as characteristic to the structure of MOF in the state of Paraná (Mognon et al., 2012; Rode et al., 2010). Our results emphasize the importance of studying MOF remnants under different environmental conditions. Araucaria angustifolia was emphasized in the study of Watzlawick et al. (2005), which was conducted in a remnant located on soils with less developed pedogenetic horizons, such as cambisols and neosols, the latter being similar to the soil found in P2.

In P3, characterized by Latosol Bruno, Araucaria angustifolia had its highest density (114 ind ha $\left.{ }^{-1}\right)$ and basal area $\left(26.25 \mathrm{~m}^{2} \mathrm{ha}^{-1}\right)$. The high value of absolute dominance shows the representativeness of the species in the MOF structure. Characteristics such as the greater effective depth of the horizons provided by pedogenetic processes favor the species predominance in this condition (Silva et al., 2001). Another important species in this pedological unit was Campomanesia xanthocarpa, which showed similar expressive VI values in sites with higher B horizon thickness such as Latosol Bruno and Haplic Cambisol (Cordeiro \& Rodrigues, 2007).

In $\mathrm{P} 4$, the hydromorphic parameter contributed to Gymnanthes klotzschiana development. This species supports a variation of water saturation and, in some cases, even forms monodominant populations (Silva et al., 2010). According to Kolb et al. (1998), Gymnanthes klotzschiana succeds in colonizing environments with higher water stress because this species presents morpho-anatomical and metabolic adaptations that allow survival in periods when soil swelling occurs due to poor drainage or elevation of water table level.

The indicator species analysis (IndVal), obtained for each site, add ecological significance to the characterization of communities, since each species is considered independently (Cáceres \& Legendre, 2009). The phytosociological values corroborated IndVal results, which reflected the specificity of species considering a given site. Species that were important to forest structure, but were not qualified as indicators, show environmental plasticity and can withstand the particular characteristics of each site. The intrinsic characteristics that allow tree species in different environmental conditions may promote occurrence in sites with different soil conditions. On the other hand, they may limit the competitive ability and restrict abundance when considered the total number of species present (Silva et al., 2016).

Our results showed correlations between species and edaphic variables such as exchangeable acidity $\left(\mathrm{H}+\mathrm{Al}^{+3}\right)$, aluminum concentration $\left(\mathrm{Al}^{+3}\right)$, organic carbon $(\mathrm{C})$ and phosphorus $(\mathrm{P})$, cation exchange capacity $(\mathrm{T})$, base saturation percentage $(\mathrm{V} \%)$, soil hydrogenation potential $\left(\mathrm{pH}\right.$ in $\left.\mathrm{CaCl}_{2}\right)$, drainage class, clay fraction and soil class. The response of plant species to physical and chemical soil variables has been previously reported in other studies. For example, Souza et al. (2012) found that the distribution of species along a topographic gradient was associated with variations in chemical fertility, acidity, and soil texture; Carvalho et al. (2005) inferred that water table level, organic matter content and $\mathrm{Al}^{+3}$ were responsible for the distribution of species in the environment; Rodrigues et al. (2007) related plant occurrences with soil fertility and texture. Our RDA results generated eigenvalues that produced short gradients of correlation between species and environmental factors. Fagundes et al. (2007) indicated that, in this case, there is a greater variation in the density of individuals than necessarily in the turnover of species composition. Our study emphasizes together with 
the aforementioned authors the importance of soil variables in structuring plant communities at local scales.

The cumulative proportion of species variance explained by soil variables showed that edaphic factors are important for clarifying local patterns of species composition; however, such factors do not account for all variance and cannot be isolated considered. Correlations between species' local distribution and environmental variables represent only part of the reality, since the density of individuals within the forest depends on a series of events (Carvalho et al., 2005) such as past anthropogenic impacts. Selective logging of arboreal species with economic value, for instance, is an important factor; however, its evidence tends to be suppressed over time, becoming difficult to be identified. Other factors such as the opening of gaps in the forest due to natural events, edge effects, trophic competition and overlapping of ecological niches interact with soil attributes to determine community structure (Pinto et al., 2008; Pontara et al., 2016; Sanchez et al., 2013).

\section{CONCLUSIONS}

Our results indicate that local abundance and distribution of species in plant communities of MOF are correlated to physical and chemical soil parameters, that change according to the predominant soil type. Environmental classification and species ordination according to edaphic gradients are important steps for understanding the distribution, occupation, and behavior of tree species in forest remnants. Moreover, we contribute to the knowledge about how species respond to different environmental conditions, which is an essential information to support forest management and restoration projects that, in many cases, lack of scientific base, leading to increased costs and lower efficiency.

\section{ACKNOWLEDGEMENTS}

The Maack family for allowing the study of the forest of “Três Capões” farm.

\section{SUBMISSION STATUS}

Received: 9 Nov. 2017

Accepted: 3 Jan. 2019

Associate editor: João Vicente de Figueiredo Latorraca

(D) 0000-0002-5969-5199

\section{CORRESPONDENCE TO}

\section{Juliano Cordeiro}

Universidade Federal do Paraná (UFPR), Rua Pioneiro, 2.153, CEP

85950-000, Palotina, PR, Brasil

e-mail: julianocordeiro@ufpr.br

\section{REFERENCES}

Anderson MJ, Crist TO, Chase JM, Vellend M, Inouye BD, Freestone AL, Sanders NJ et al. Navigating the multiple meanings of beta diversity: a roadmap for the practicing ecologist. Ecology Letters 2011; 14(1): 19-28. 10.1111/j.1461-0248.2010.01552.x

Barddal ML, Roderjan CV, Galvão F, Curcio GR. Caracterização florística e fitossociológica de um trecho sazonalmente inundável de floresta aluvial, em Araucária, PR. Ciência Florestal 2004; 14(2): 37-50. 10.5902/198050981805

Cáceres M, Legendre P. Associations between species and groups of sites: indices and statistical inference. Ecology 2009; 90(12): 35663574. 10.1890/08-1823.1

Camargos VL, Silva FS, Meira Neto JAA, Martins SV. Influência de fatores edáficos sobre variações florísticas na floresta estacional semidecídua no entorno da Lagoa Carioca, Parque Estadual do Rio Doce, MG, Brasil. Acta Botanica Brasilica 2008; 22(1): 75-84. 10.1590/S0102-33062008000100010

Carvalho DA, Oliveira-Filho AT, Van Den Berg E, Fontes MAL, Vilela EA, Marques JJGS, Carvalho WAC. Variações florísticas e estruturais do componente arbóreo de uma Floresta Ombrófila Alto-Montana às margens do rio Grande. Bocaina de Minas, MG, Brasil. Acta Botanica Brasilica 2005; 19(1): 91-100. 10.1590/S010233062005000100010

Carvalho J, Marques MCM, Roderjan CV, Barddal M, Sousa SGA. Relações entre a distribuição das espécies em diferentes estratos e as características do solo em uma floresta aluvial no estado do Paraná, Brasil. Acta Botanica Brasilica 2009; 23(1): 1-9. 10.1590/ S0102-33062009000100002

Castella PR, Britez RM. A floresta com Araucária no Paraná: conservação e diagnóstico dos remanescentes florestais. Brasília: Ministério do Meio Ambiente; 2004.

Cordeiro J, Rodrigues WA. Caracterização fitossociológica de um remanescente de Floresta Ombrófila Mista em Guarapuava, PR. Revista Árvore 2007; 31(3): 545-554. 10.1590/S010067622007000300020

Curcio GR, Bonnet A, Pestana D, Souza L, Socher LG, Galvão F, Roderjan CV. Compartimentação topossequencial e caracterização fitossociológica de um capão de Floresta Ombrófila Mista. Revista Floresta 2006; 36(3): 361-369. 10.5380/rf.v36i3.7515

Curcio GR, Galvão F, Bonnet A, Barddal ML, Dedecek RA. A floresta fluvial em dois compartimentos do Rio Iguaçu, Paraná, Brasil. Revista Floresta 2007; 37(2): 125-146. 10.5380/rf.v37i2.8645

Dufrêne $M$, Legendre $P$. Species assemblages and indicator species: the need for a flexible asymmetrical approach. Ecological Monographs 1997; 67(3): 345-366. 10.1890/0012-9615(1997)067[0345:SAAIST]2.0.CO;2

Empresa Brasileira de Pesquisa Agropecuária - Embrapa. Sistema brasileiro de classificação de solos. 3rd ed. Brasília: Embrapa Produção de Informação; 2013.

Fagundes LM, Carvalho DA, Van Den Berg E, Marques JJGSM, Machado LM. Florística e estrutura do estrato arbóreo de dois fragmentos de florestas decíduas às margens do rio Grande, em Alpinópolis e Passos, MG, Brasil. Acta Botanica Brasilica 2007; 21(1): 65-78. 10.1590/S0102-33062007000100007 
Fundação de Pesquisas Florestais do Paraná - FUPEF. Conservação do bioma floresta com Araucária: relatório final: diagnóstico dos remanescentes florestais. Curitiba: FUPEF; 2001.

Hirst CN, Jackson DA. Reconstructing community relationships: the impact of sampling error, ordination approach, and gradient length. Diversity and Distributions 2007; 13(4): 361-371. 10.1111/j.14724642.2007.00307.x

Instituto Brasileiro de Geografia e Estatística - IBGE. Manual técnico da vegetação brasileira. Rio de Janeiro: IBGE; 2012. (Série Manuais Técnicos em Geociências; 1).

Jardim Botânico do Rio de Janeiro - JBRJ. Flora do Brasil $2020 \mathrm{em}$ construção [Internet]. [2018?] [cited 2018 Oct. 15]. Available from: https://bit.ly/33lexMg

Kolb RM, Medri ME, Bianchini E, Pimenta JA, Giloni PC, Correa GT. Anatomia ecológica de Sebastiania Commersoniana (Baillon) Smith e Downs (Euphorbiaceae) submetida ao alagamento. Revista Brasileira de Botânica 1998; 21(3): 305-312. 10.1590/S010084041998000300010

Legendre P, Legendre L. Numerical ecology. 3rd ed. Amsterdam: Elsevier; 2012.

Lortie CJ, Brooker RW, Choler P, Kikvidze Z, Michalet R, Pugnaire FI, Callaway RM. Rethinking plant community theory. Oikos 2004; 107(2): 433-438. 10.1111/j.0030-1299.2004.13250.x

Marcilio-Silva V, Zwiener VP, Marques MCM. Metacommunity structure, additive partitioning and environmental drivers of woody plants diversity in the Brazilian Atlantic Forest. Diversity and Distributions 2017; 23(10): 1110-1119. 10.1111/ddi.12616

Minerais do Paraná S.A. - Mineropar. Atlas geológico do estado do Paraná. Curitiba: Mineropar; 2001.

Minerais do Paraná S.A. - Mineropar. Atlas geomorfológico do estado do Paraná. Curitiba: Mineropar; 2006.

Mittermeier RA, Turner WR, Larsen FW, Brooks TM, Gascon C. Global biodiversity conservation: the critical role of hotspots. In: Zachos FE, Habel JC, editors. Biodiversity Hotspots: distribution and protection of conservation priority areas. Heidelberg: Springer-Verlag Berlin; 2011. p. 3-22.

Mognon F, Dallagnol F, Sanquetta C, Corte AP, Maas G. Uma década de dinâmica florística e fitossociológica em Floresta Ombrófila Mista Montana no sul do Paraná. Revista de Estudos Ambientais 2012; 14(1): 43-59. 10.7867/1983-1501.2012v14n1p43-59

Mueller-Dumbois D, Ellenberg H. Aims and methods vegetation ecology. New York: John Wiley \& Sons; 1974.

Munhoz HM. Localization map of the "Três Capões" farm, Guarapuava, Southern Brazil. Trabalhos técnicos. [Place unknown]; 2018. B\&W, $20 \times 30 \mathrm{~cm}, 1 \mathrm{p}$.

Oliveira-Filho AT, Curi N, Vilela EA, Carvalho DA. Variation in tree community composition and structure with changes in soil properties within a fragment of semideciduous forest in southeastern Brazil. Edinburgh Journal of Botany 2001; 58(1): 139-158. 10.1017/S0960428601000506

Peel MC, Finlayson BL, McMahon TA. Updated world map of the Köppen-Geiger climate classification. Hydrology and Earth System Sciences 2007; 11: 1633-1644. 10.5194/hess-11-1633-2007
Pinto JRR, Oliveira-Filho AT, Hay JDV. Influence of soil and topography on the composition of a tree community in a central Brazilian valley forest. Edinburgh Journal of Botany 2005; 62(1-2): 69-90. 10.1017/S0960428606000035

Pinto SIC, Martins SV, Barros NF, Dias HCT, Kunz SH. Influence of environmental variables on the shrub and tree species distribution in two Semideciduous Forest sites in Viçosa, Minas Gerais, Brazil. Revista de Biología Tropical 2008; 56(3): 1557-1569. 10.15517/rbt. v56i3.5729

Pontara V, Bueno ML, Garcia LE, Oliveira-Filho AT, Pennington TR, Burslem DFRP, Lemos-Filho JP. Fine-scale variation in topography and seasonality determine radial growth of an endangered tree in Brazilian Atlantic forest. Plant Soil 2016; 403(1-2): 115-128. 10.1007/ s11104-016-2795-3

R Core Team. $R$ : A language and environment for statistical computing. Vienna: R Foundation for Statistical Computing; 2016.

Reinhart KO. The organization of plant communities: negative plant-soil feedbacks and semiarid grasslands. Ecology 2012; 93(11): 2377-2385. 10.1890/12-0486.1

Resende M, Lani JL, Rezende SB. Pedossistemas da Mata Atlântica: considerações pertinentes sobre a sustentabilidade. Revista Árvore 2002; 26(3): 261-269. 10.1590/S0100-67622002000300001

Rodal MJN, Sampaio EVSB, Figueiredo MA, editors. Manual sobre métodos de estudos florístico e fitossociológico: ecossistema Caatinga. Brasília: Sociedade Botânica do Brasil; 2013.

Rode R, Figueiredo Filho A, Galvão F, Machado SA. Estrutura horizontal da comunidade arbórea sob um povoamento com Araucaria angustifolia e uma Floresta Ombrófila Mista. Pesquisa Florestal Brasileira 2010; 30(64): 347-361. 10.4336/2010.pfb.30.64.347

Rodrigues LA, Carvalho DA, Oliveira-Filho AT, Curi N. Efeitos de solos e topografia sobre a distribuição de espécies arbóreas em um fragmento de floresta estacional semidecidual em Luminárias, MG. Revista Árvore 2007; 31(1): 25-35. 10.1590/S010067622007000100004

Sanchez M, Pedroni F, Eisenlohr PV, Oliveira-Filho AT. Changes in tree community composition and structure of Atlantic rainforest on a slope of the Serra do Mar range, southeastern Brazil, from near sea level to $1000 \mathrm{~m}$ of altitude. Flora 2013; 208(3): 184-196. 10.1016/j.flora.2013.03.002

Silva AC, Higuchi P, van den Berg E. Effects of soil water table regime on tree community species richness and structure of alluvial forest fragments in southeast Brazil. Brazilian Journal of Biology 2010; 70(3): 465-471. 10.1590/S1519-69842010000300002

Silva HD, Bellote AFJ, Ferreira CA, Bognola IA. Recomendação de solos para Araucaria angustifolia com base nas suas propriedades físicas e químicas. Boletim de Pesquisas Florestais 2001; (43): 61-74.

Silva JO, Silva AC, Higuchi P, Mafra AL, Loebens R, Rodrigues LC Jr et al. Heterogeneidade ambiental e regeneração natural em uma Floresta Ombrófila Mista Aluvial. Scientia Forestalis 2016; 44(112): 787-797. 10.18671/scifor.v44n112.01

Sollins P. Factors influencing species composition in tropical lowland rain forest: does soil matter? Ecology 1998; 79(1): 23-30. $10.2307 / 176861$

Souza PB, Lelis JJ, Schaefer CEG, Souza AL, Meira Neto JAM. Distribution of tree species in a geomorphological and pedological 
gradient of Submontane Semidecidual Seasonal Forest in the vicinity of Rio Doce State Park, Minas Gerais. Revista Árvore 2012; 36(4): 707-718. 10.1590/S0100-67622012000400012

Ter Braak CJF, Prentice IC. A theory of gradient analysis. Advances in Ecological Research 2004; 34: 235-282. 10.1016/S00652504(03)34003-6

Vibrans AC, Uhlmann A, Sevegnani L, Marcolin M, Nakajima N, Grippa CR et al. Ordenação dos dados de estrutura da Floresta Ombrófila Mista partindo de informações do inventário florístico- florestal de Santa Catarina: resultados de estudo-piloto. Revista Ciência Florestal 2008; 18(4): 511-523. 10.5902/19805098434

Watzlawick LF, Sanquetta CR, Valério AF, Silvestre R. Caracterização da composição florística e estrutura de uma Floresta Ombrófila Mista no município de General Carneiro (PR). Ambiência 2005; 1(2): 229-237.

Zwiener VP, Padial AA, Marques MCM, Faleiro FV, Loyola R, Peterson AT. Planning for conservation and restoration under climate and land use change in the Brazilian Atlantic Forest. Diversity and Distribution 2017; 23(8): 955-966. 10.1111/ddi.12588 